\title{
A phase I safety and efficacy study of the mammary aspirate specimen cytology test device for collection of specimens for exfoliative cytopathology of the breast ducts ${ }^{*}$
}

\author{
Shu-Chih Chen ${ }^{1,2}$, Stephen A. Vitkun ${ }^{3}$, Steven C. Quay ${ }^{1,2 \#}$ \\ ${ }^{1}$ Atossa Genetics, Inc., Seattle, USA \\ ${ }^{2}$ The National Reference Laboratory for Breast Health, Inc., Seattle, USA \\ ${ }^{3}$ Department of Anesthesiology, State University of New York at Stony Brook, Stony Brook, USA \\ Email: "Steven.Quay@atossagenetics.com
}

Received 19 June 2013; revised 20 July 2013; accepted 10 August 2013

Copyright (c) 2013 Shu-Chih Chen et al. This is an open access article distributed under the Creative Commons Attribution License, which permits unrestricted use, distribution, and reproduction in any medium, provided the original work is properly cited.

\begin{abstract}
Background: The ability to identify asymptomatic women at high risk for breast cancer using known pre-malignant changes in exfoliative cytopathology of nipple aspirate fluid is of clinical importance. Exfoliative cytopathology of Nipple Aspirate Fluid (NAF) has been shown to be an important adjunct to the currently accepted standard of medical care, i.e. mammography, coupled with physical examination, for the diagnosis of breast cancer. This is especially important for the subset of women aged 18 - 50 who are not identified as "high risk", and therefore, for whom mammography is not routinely recommended. The objective of this study was to determine if a new, patented Class II medical device, the Mammary Aspirate Specimen Cytology Test (MASCT) System, designed to collect NAF for subsequent cytological examination is safe and effective. Methods: The MASCT medical device is a modified breast pump and was used to obtain bilateral specimens from 34 healthy, non-pregnant, female subjects for cytopathological examination. A conventional breast disease work-up was performed (medical history/risk factor collection, clinical breast examination and mammogram) and NAF specimens were collected. Specimen weight was measured and a cytopathological examination was performed. Vital signs measurements, clinical laboratory analysis, and adverse event reporting were performed. Results: Based on cytopathological evaluation and/or measurable weight changes on the specimen collection membrane filter, all breasts evaluated
\end{abstract}

*Conflicts of interest: Shu-Chih Chen and Steven Quay are employees and have an equity interest in Atossa Genetics, Inc. Stephen Vitkun declares no conflicts of interest.

\#Corresponding author.
(100\%) yielded nipple aspirate fluid. Specimen weights ranged from $<1$ to $37 \mathrm{mg}$ and all specimens evaluated cytopathologically were deemed to be clinically useful. One patient's specimen was not available for cytopathological examination due to specimen mishandling, resulting in 60 breasts (representing 30 subjects) being evaluated cytologically. Fifty-eight of sixty breasts evaluated cytopathologically $(97 \%)$ were reported as cytology Class I, and 2 of $60(3 \%)$ were reported as cytology Class IIa. Cytopathological findings correlated well with mammogram and clinical breast exam results. No adverse events, including pain from the collection procedure, were reported. Conclusion: Based on this clinical study, we conclude that the Mammary Aspiration Specimen Cytology Test device is safe and effective for the collection of mammary aspirate specimens for laboratory cytopathological testing.

Keywords: Breast Cancer Risk; Atypical Hyperplasia; Exfoliative Cytopathology; Phase I Clinical Trial; Nipple Aspirate Fluid

\section{INTRODUCTION}

Breast cancer is the most common cancer and a leading cause of cancer deaths among women in the United States. Two hundred thirty-two thousand women in the US are diagnosed each year with this disease. Prevention efforts are hindered by the fact that analysis of the tissue at risk traditionally requires a surgical biopsy. A non-operative method to evaluate women at high risk for breast cancer, women with known pre-malignant changes in their breasts, or to identify pre-malignant changes in asymptomatic women would be very beneficial. Mam- 
mary (nipple) aspiration provides a non-surgical method of obtaining breast intraductal epithelial cells, where over $95 \%$ of breast cancer arises, and has been performed successfully in tens of thousands of women.

While mammary aspiration has been performed in many women, most of the women who underwent the procedure were of normal breast cancer risk, and the success in obtaining intraductal fluid has historically been well below 50\% [1]. Furthermore, intraductal fluid containing sufficient epithelial cells to evaluate biomarkers has been obtained in only approximately $20 \%$ of individuals [2]. In contrast, fine needle aspiration or core biopsy, commonly using clinical tools to diagnose a mammographic or palpable abnormality in the breast, reports greater success rates but is an obviously invasive process.

The overall goal of this product development is FDA market clearance for the following indication:

The MASCT device is intended for use in the collection of nipple aspirate fluid for cytological testing. The collected fluid can be used in the determination and/or differentiation of normal versus premalignant versus malignant cells.

\section{BACKGROUND}

Mammary aspiration is a non-operative, non-invasive biopsy technique to obtain exfoliated breast intraductal epithelium as well as resident cells within the ductlobular system.

Mammary Aspiration Specimen (MAS) Technique: MAS technique uses a modified breast pump to obtain intraductal fluid, containing intraductal epithelial cells, for cytopathological examination. The negative pressure produced has been likened to that produced by a suckling baby and has been well tolerated by all women who participated in this clinical trial. In addition, the mammary aspiration technique has been employed using similar devices without significant side effects in over 14,000 women. Success rates for these other devices have been reported as below 50\% [1].

Mammary Aspirate Specimen (MAS) Cytology: MAS specimens contain foam cells, apocrine metaplastic cells, lymphocytes, and exfoliated ductal epithelial cells. Epithelial atypia and hyperplasia in MAS are the cytopathological equivalents of histologic epithelial atypical hyperplasia and hyperplasia without atypia (usual ductal hyperplasia), respectively [2].

OBJECTIVE: The objective of this study was to determine if a new, patented device (the MASCT System), designed to collect MAS for subsequent cytological examination, is safe and effective.

\section{METHODS}

\subsection{Study Design}

This was a single-center study that involved healthy, non-pregnant, female subjects. Subjects were enrolled in order of appearance at the clinic. A total of 34 subjects were screened and all 34 subjects were enrolled. All subjects were screened and evaluated according to the following criteria:

Inclusion Criteria:

1) Healthy, female, age 18 - 73 years and within $\pm 25 \%$ of ideal body weight (according to the Metropolitan Life Table);

2) Non-lactating and non-pregnant (as documented by a negative urine pregnancy test on the day of study);

3) Good general health as determined by medical history, breast disease/cancer history, clinical breast exam, mammography, vital signs, and blood and urine laboratory tests;

4) Willing to give informed consent and follow study procedures as directed;

5) Negative test for drugs of abuse documented on the day of the study.

Exclusion Criteria:

1) Medical condition/psychiatric problems making subjects a poor candidate for study, as determined by the Principal Investigator;

2) Pregnancy or suspicion of pregnancy;

3) Participation in an investigational drug or device study less than 30 days prior to enrollment in this study;

4) Pregnancy, childbirth, or lactation less than 90 days prior to enrollment in this study;

5) Acute illness, including taking antibiotics, analgesics, antipyretics and/or cold medications less than 7 days prior to enrollment in this study.

Efficacy observations consisted of a conventional breast health work-up (medical history/risk factor collection, clinical breast examination [3] and mammogram [4]), and collecting mammary aspiration specimens (nipple aspiration fluid) from women, weighing the specimens, and evaluating them for cellularity and cytological diagnosis. Safety observations consisted of vital signs, clinical laboratory analysis, and adverse event reporting.

The study was conducted from a pre-determined protocol, which was reviewed and approved for the use of human subjects by an institutional review board, Quorum Review, Seattle, Washington.

\subsection{MAS Collection}

\subsubsection{The Device and Kit}

The device is a patented modified breast pump in design, permitting the aspiration and collection of picoliter quantities of fluid and designed to be conveniently operated with one hand (Figure 1). The pump is constructed principally of polycarbonate and generates between 150 and $200 \mathrm{~mm} \mathrm{Hg}$ negative pressure, the range that maximizes milk yield in nursing mothers [5]. The specimen collection membrane is constructed of a membrane filter 


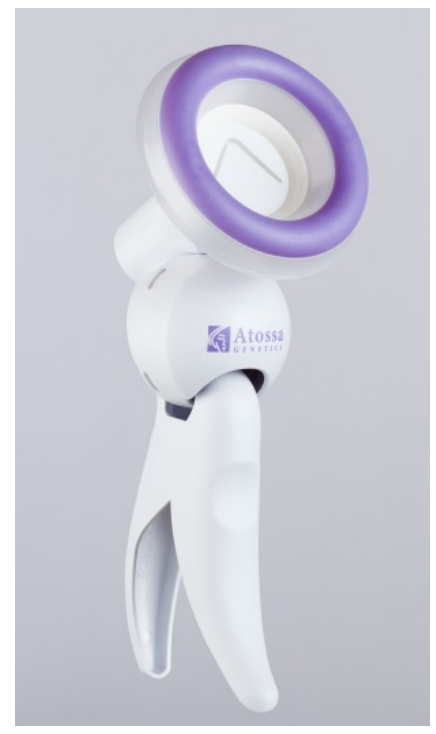

Figure 1. The MASCT System includes the modified, hand-held breast pump and two membrane collection units.

(Marion Chicago, West Chicago, IL).

The collection funnel is cone shaped and is designed to fit over the breast's nipple-areolar area. At the lower end of the collection funnel there is a Nipple Aspirate Fluid (NAF) specimen collection device (the "flower") which is composed of a polycarbonate frame and a membrane filter. Immediately behind that in the assembled device is the vented specimen collection vial into which excess specimen can collect.

\subsubsection{The Procedure}

Subjects were placed in a recumbent position and the nipple and surrounding areola of both breasts were cleansed with Nu-Prep skin prepping gel. This removed the keratin plugs at the end of the milk ducts. An alcohol wipe to clean the nipple and periareolar area could be used instead of Nu-Prep, taking care to let the alcohol on the nipple dry completely before moving onto the next step. A warm moist cloth or heating pad was placed on both breasts for 10 minutes. The subjects were then placed in the sitting position for the aspiration step. The right breast was aspirated first. The pad was removed and the device was placed over the areola. The subject was asked to compress her breast with both hands while the breast pump was actuated for 90 -seconds by the physician's rhythmically squeezing of the pump's hand lever to mimic the sinusoidal negative pressure change that occurs during suckling. Specimen fluid, in the form of droplets, was collected on the tarred filter disks. The quantity of NAF was expected to vary from $<1-100 \mu \mathrm{l}$ [1] (equivalent to $<1$ to $100 \mathrm{mg}$ weight change). The negative pressure produced was well tolerated. The same procedure was then performed on the left breast.

Once the NAF was collected from both breasts, the specimens were weighed and recorded. The specimens were then refrigerated and sent to the laboratory for cytological analysis.

\subsection{Cytology Preparation}

The NAF specimens were weighed and then the filters were processed for general cytology.

\subsection{Cytopathology Interpretation}

A trained, board-certified cytopathologist read the slides. The data was reported in the case report form according to the format recommended by the College of American Pathologists [6]. The criteria for reading and interpreting the slides are contained in Table $\mathbf{1}$ as adapted from King, et al. [7].

\subsection{Statistical Analysis}

Summary efficacy statistics in the form of specimen size, mean, standard deviation, median, minimum and maximum are presented for the weight of Mammary Aspiration Specimen (MAS). The primary objective of this study was addressed by assessing the weight of MAS and cytological findings for each breast evaluated.

The number and percentage of women that produced measurable fluid was calculated (see Table 3). A table was generated comparing the results of the clinical breast examination (CBE), the mammogram (M), and the cytology (C) evaluation. The three classes of the CBE are normal/no findings, normal/findings, and abnormal/ findings. See Table 2 for the BI-RADS [4] classification of mammograms.

The safety observations were the mammogram, the diagnostic cytology examination of the specimens, the clinical laboratory analytes measured before the procedure, the pain associated with the procedure, and the clinical adverse event reporting. The mammogram and cytopathology examination were recorded. The clinical laboratory analytes have been tabulated individually and presented in data listings in Table 3.

\section{RESULTS}

\subsection{Efficacy}

The primary efficacy objectives were to determine whether the device could collect specimens and whether the collected specimens had diagnostic utility.

Based on cytopathological evaluation and/or measureable weight changes on the specimen collection membrane filter, all breasts evaluated (100\%) with the mammary aspiration technique using the MASCT System 
Table 1. Cytopathological criteria for mammary aspirate specimens.

\begin{tabular}{|c|c|c|}
\hline Class & Finding & Description \\
\hline $\mathbf{I}$ & Scant mammary epithelial cells & $\begin{array}{l}\text { Includes acellular specimens, those with only foam cells, and those with fewer than five } \\
\text { mammary cells }\end{array}$ \\
\hline IIa & $\begin{array}{l}\text { Benign mammary epithelial cells } \\
\text { a) Normal/non-papillary }\end{array}$ & $\begin{array}{l}\text { Consists of specimens containing more than five mammary epithelial cells without } \\
\text { cytological atypia. This category encompasses normal mammary cells and apocrine } \\
\text { metaplasia, duct hyperplasia with atypia. } \\
\text { Normal/non-papillary: single cells or small, loosely cohesive aggregates of cells }\end{array}$ \\
\hline IIb & $\begin{array}{l}\text { Benign mammary epithelial cells } \\
\text { b) Hyperplastic }\end{array}$ & $\begin{array}{l}\text { Consists of specimens containing more than five mammary epithelial cells without } \\
\text { cytological atypia. This category encompasses normal mammary cells and apocrine } \\
\text { metaplasia, duct hyperplasia with atypia. } \\
\text { Hyperplastic: large 3-dimensional clusters of cells (indicative of duct hyperplasia or } \\
\text { papillomatosis) }\end{array}$ \\
\hline III & Atypical mammary epithelial cells & $\begin{array}{l}\text { Specimens with five or more mammary epithelial cells that exhibit atypical features. Atypia } \\
\text { is defined as nuclear enlargement or a chromatin distribution abnormally short of obvious } \\
\text { malignant criteria. Notation should be made whether the atypical cells are single or in } \\
\text { papillary clusters. }\end{array}$ \\
\hline IV & Malignant cells present & $\begin{array}{l}\text { Specimens that contain cells with unequivocal criteria of malignancy. If the malignant cells } \\
\text { are fewer than five, the specimens are designated "scanty evidence". }\end{array}$ \\
\hline
\end{tabular}

Table 2. Mammography assessment categories (BI-RADS).

\begin{tabular}{ccc}
\hline Category & Finding & Assessment \\
\hline $\mathbf{0}$ & AI & Assessment Incomplete, need additional imaging evaluation \\
$\mathbf{1}$ & $\mathbf{N}$ & Negative \\
2 & BF & Benign Finding \\
3 & PBF & Probably Benign Finding, short interval follow-up suggested \\
$\mathbf{4}$ & SA & Suspicious Abnormality, biopsy should be considered \\
$\mathbf{5}$ & HM & Highly Suggestive of Malignancy, appropriate action should be taken \\
\hline
\end{tabular}

device yielded nipple aspirate fluid. Specimen weights ranged from $<1$ to $37 \mathrm{mg}$ and all specimens evaluated cytologically were deemed to be clinically useful.

There was a protocol deviation related to specimen handling. The specimens for one subject (\#07BP) were inadvertently frozen, causing them to be unsuitable for cytopathological evaluation. This resulted in 30 subjects (60 breasts) evaluated for cytopathology. It should be noted that this patient was included in the safety analysis and the weight of NAF specimens were included in the analysis thereof.

The mean mammary aspiration specimen weight was $6.6 \mathrm{mg}$, with a range of $<1$ to $37 \mathrm{mg}$. While $13 \%$ of the specimens were reported as $<1 \mathrm{mg}$ due to weighing variability from the small volume of the specimens, all of the specimens were sufficient for cytopathological analysis in that the pathologist was able to read all of the specimens and cytopathology results were obtained. Fiftyeight of sixty specimens or $97 \%$ were Class I, and two of 60 (3\%) were Class IIa. Both Class IIa findings were benign.

Table 3 lists data comparing the cytopathology (MAS) results with the mammogram $(\mathrm{M})$ and the clinical breast examination (CBE). There was general correlation of the MAS findings with both the CBE and the $\mathrm{M}$.

On CBE, 52 of the 62 breasts examined were reported as "normal/no findings"; eight were reported as "normal/ findings" and two reported as "abnormal/findings". It should be noted that the two observations of "abnormal/findings" were due to the subject's having breast implants and no other tissue-related findings were reported for this subject. The eight reports of "normal/ findings" on CBE were all related to fibrocystic breasts. Therefore, it can be concluded that no clinically significant findings were reported on CBE.

Mammograms were performed on all 62 breasts and of these, 38 reported negative results, 15 reported benign findings (e.g. small cysts, nodules, lymph nodes, or dense breast parenchyma) and nine reported probably benign findings (e.g. calcifications, nodules, or dense breast parenchyma), defined as findings that were of no clinical concern at this time but which warranted additional follow-up in the short term (6 - 12 months). In one subject, the mammogram reported calcifications in both breasts that were classified as probably benign findings requiring follow-up. The MAS result for this subject's 
Table 3. Efficacy data—comparison of clinical breast exam (CBE), mammogram (M), and cytopathology (MAS).

\begin{tabular}{|c|c|c|c|c|c|c|}
\hline \multirow[b]{2}{*}{ Subject $^{\#}$} & \multicolumn{3}{|c|}{ Right Breast } & \multicolumn{3}{|c|}{ Left Breast } \\
\hline & CBE & $\mathbf{M}$ & MAS & CBE & $\mathbf{M}$ & MAS \\
\hline 01BP & Normal/No Findings & $\mathrm{BF}$ & I & Normal/No Findings & $\mathrm{BF}$ & I \\
\hline 02BP & Normal/Findings & $\mathrm{N}$ & I & Normal/Findings & $\mathrm{N}$ & I \\
\hline 03BP & Normal/Findings & $\mathrm{BF}$ & I & Normal/Findings & $\mathrm{BF}$ & I \\
\hline 04BP & Normal/Findings & $\mathrm{N}$ & I & Normal/Findings & $\mathrm{N}$ & I \\
\hline 05BP & Normal/No Findings & $\mathrm{N}$ & I & Normal/No Findings & $\mathrm{N}$ & I \\
\hline 06BP & Normal/No Findings & $\mathrm{N}$ & I & Normal/No Findings & $\mathrm{N}$ & I \\
\hline 07BP & Normal/No Findings & PBF & $\mathrm{n} / \mathrm{a}^{1}$ & Normal/No Findings & $\mathrm{PBF}$ & $\mathrm{n} / \mathrm{a}^{1}$ \\
\hline 08BP & Normal/No Findings & PBF & I & Normal/No Findings & PBF & I \\
\hline 09BP & Normal/Findings & $\mathrm{BF}$ & I & Normal/Findings & $\mathrm{BF}$ & I \\
\hline 10BP & Normal/No Findings & $\mathrm{N}$ & I & Normal/No Findings & $\mathrm{N}$ & I \\
\hline $11 \mathrm{BP}$ & Normal/No Findings & $\mathrm{N}$ & I & Normal/No Findings & $\mathrm{N}$ & I \\
\hline $12 \mathrm{BP}$ & Normal/No Findings & $\mathrm{N}$ & I & Normal/No Findings & $\mathrm{N}$ & I \\
\hline 13BP & Normal/No Findings & $\mathrm{N}$ & I & Normal/No Findings & $\mathrm{N}$ & I \\
\hline 14BP & Normal/No Findings & $\mathrm{N}$ & I & Normal/No Findings & $\mathrm{N}$ & I \\
\hline $15 \mathrm{BP}$ & Normal/No Findings & $\mathrm{BF}$ & I & Normal/No Findings & $\mathrm{BF}$ & I \\
\hline 16BP & Normal/No Findings & $\mathrm{N}$ & I & Normal/No Findings & $\mathrm{N}$ & I \\
\hline 17BP & Normal/No Findings & $\mathrm{N}$ & I & Normal/No Findings & $\mathrm{N}$ & I \\
\hline 18BP & Normal/No Findings & $\mathrm{N}$ & I & Normal/No Findings & $\mathrm{N}$ & I \\
\hline 19BP & Normal/No Findings & $\mathrm{N}$ & I & Normal/No Findings & $\mathrm{N}$ & I \\
\hline 20BP & Normal/No Findings & PBF & IIa & Normal/No Findings & PBF & I \\
\hline $21 \mathrm{BP}^{\ddagger}$ & Abnormal/Findings & $\mathrm{BF}$ & I & Abnormal/Findings & $\mathrm{BF}$ & I \\
\hline 23BP & Normal/No Findings & $\mathrm{N}$ & I & Normal/No Findings & $\mathrm{N}$ & I \\
\hline 24BP & Normal/No Findings & $\mathrm{N}$ & IIa & Normal/No Findings & $\mathrm{N}$ & I \\
\hline $25 \mathrm{BP}$ & Normal/No Findings & $\mathrm{N}$ & I & Normal/No Findings & $\mathrm{N}$ & I \\
\hline 26BP & Normal/No Findings & $\mathrm{N}$ & I & Normal/No Findings & $\mathrm{N}$ & I \\
\hline $27 \mathrm{BP}$ & Normal/No Findings & PBF & I & Normal/No Findings & $\mathrm{N}$ & I \\
\hline 28BP & Normal/No Findings & $\mathrm{N}$ & I & Normal/No Findings & $\mathrm{BF}$ & I \\
\hline 29BP & Normal/No Findings & $\mathrm{N}$ & I & Normal/No Findings & $\mathrm{N}$ & I \\
\hline $30 \mathrm{BP}^{\ddagger}$ & Normal/No Findings & $\mathrm{BF}$ & I & Normal/No Findings & $\mathrm{N}$ & I \\
\hline 33ВР & Normal/No Findings & $\mathrm{BF}$ & I & Normal/No Findings & $\mathrm{BF}$ & I \\
\hline 34BP & Normal/No Findings & PBF & I & Normal/No Findings & PBF & I \\
\hline
\end{tabular}

${ }^{1} \mathrm{n} / \mathrm{a}=$ results not available due to specimen mishandling Mammogram: $\mathrm{N}=$ Negative; BF = Benign Findings; PBF = Probably Benign Findings; ${ }^{\ddagger}$ Indicates break in numbering sequence.

right breast was Class IIa, indicating the presence of benign ductal cells, which correlates with the mammogram findings.

Cells of ductal origin were obtained in 20 of the 30 subjects. Five cell types were identified in the MAS specimens: epithelial cells, usually ductal and rarely apocrine, foam cells, histiocytes, lymphocytes, and neutrophils. In addition, necrotic cells of uncertain origin were also found.

Ductal epithelial cells (Figure 2) were typically 10 to
15 microns in diameter but may be as large as $30 \mathrm{mi}-$ crons. The cytoplasm was cyanophilic and was either homogeneous or contained vacuoles that vary in size and number. The nuclear to cytoplasm ratio was high, with the nucleus occupying one-half to two-thirds of the cell. It is usually oval, but may be round or indented. The chromatin was fine, uniform, compact, and dark, and was arranged as a thin, uniform membrane deposit at the nuclear border. There was usually a distinct nucleolus.

Ductal cells often can be present not as single cells but 
as groups of cells (Figure 3). In one analysis of specimens from non-pregnant patients, nine (34\%) had single duct cells and seventeen (66\%) had groups of cells [1]. Cell groups were usually tightly arranged, show knobby borders where individual cells are protruding beyond the main mass, and have overlapping and multilayering centrally. They are interpreted as surface epithelium splitting from underlying cells and rolling up in the ductal secretions to form discrete groupings.

Apocrine metaplastic epithelial cells were usually two to three times the diameter of ductal epithelial cells, and have very dense, sometimes distinctly granular, basophilic or amphophilic cytoplasm and central or eccentric nuclei. The nuclear diameter was approximately one fifth to one third that of the cell cytoplasm; the nucleus is usually round, but may be oval or bean-shaped. A prominent, uniform, chromatinic membrane at the nuclear

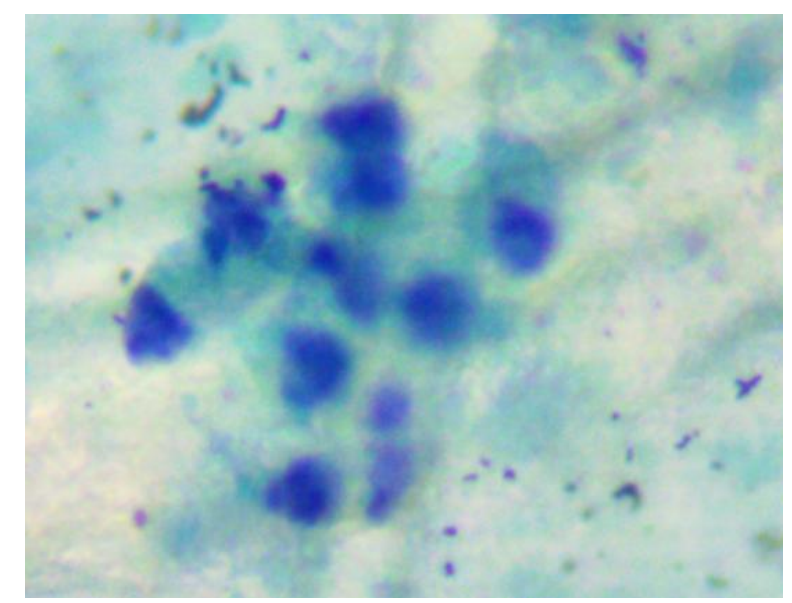

Figure 2. Clustering of ductal cells $(\times 400)$.

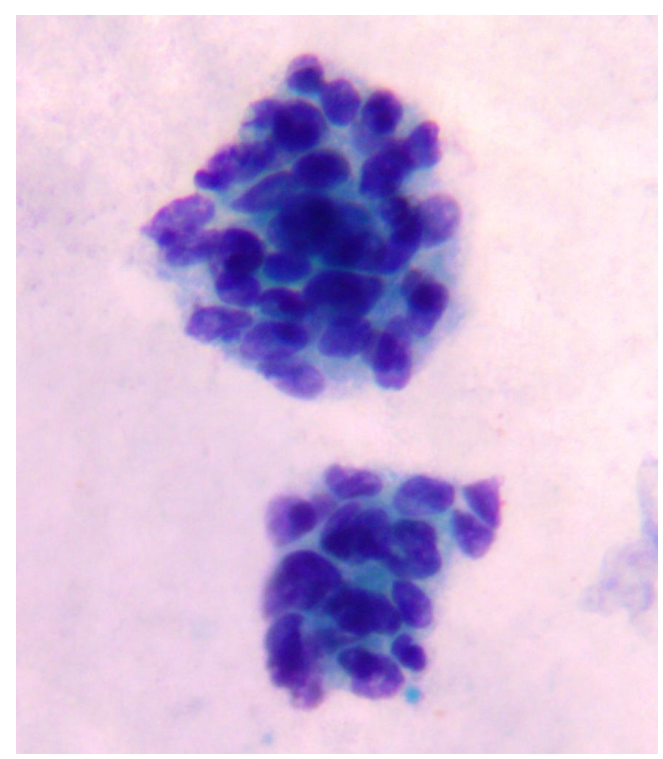

Figure 3. Multicellular clusters of atypical ductal cells $(\times 400)$. border, fine granular chromatin, and a prominent nucleolus distinguishes it. Apocrine metaplastic cells appeared singly or in clusters.

Foam cells tended to be larger than epithelial or apocrine cells, ranging from 15 to 50 microns or larger. Cytoplasm was abundant, finely vacuolated, and cyanophilic. The nucleus was usually small and round with distinct fine chromatin and parachromatin and a prominent nucleolus. The nuclear borders are smooth and accentuated by a distinct chromatinic membrane. Hyperchromasia and pyknoses are not uncommon.

Foam cells comprise a large proportion of the benign cells found in mammary aspirate specimens. It is thought that they represent a degenerate form of ductal epithetlium and some studies suggest that cytopathological examination of foam cells can provide useful information about epithelial changes [1].

Histiocytes, lymphocytes, and neutrophils are commonly found in mammary aspirates; their histological appearance is not remarkably different here than in other specimens.

\subsection{Safety}

Vital sign measurements were made and were unremarkable either before or after the procedure. The subjects were healthy upon entering the study, had no complaints or unusual findings during the course of their breast examinations, and were healthy at the close of the study. There were no clinically significant findings in the clinical laboratory analysis (see data listings in Table 3). No adverse events, including pain from the collection procedure, were reported by any of the 34 subjects.

\section{DISCUSSION}

In this study, the Mammary Aspiration Specimen Cytology Test (MASCT) System biopsy device was evaluated in a clinical setting for use in the collection of mammary aspirate specimens for laboratory exfoliative cytological testing. The results demonstrate good patient and physician acceptance of the mammary aspiration technique. The amount of time a physician or nurse would spend on the procedure was confirmed as approximately 15 minutes. There were no adverse events reported by the subjects and no safety observations of any kind. The collection process was not reported to be painful by any subject.

The cytopathology results showed that specimens were collected from all subjects who participated in the study and that the specimens made available for cytopathologic examination were adequate for examination by the pathologist for classification. A combination of specimen weights and cytologic evaluations demonstrated that specimens were collected from all 34 subjects; however, 
one subject's specimens were not available for cytologic evaluation because of laboratory mishandling. It should be noted that this subject (\#07BP) reported 15 and $6 \mathrm{mg}$ specimen weights for the right and left breast, respecttively. As stated above, because of laboratory mishandling the cytopathological examination could not be performed for this subject but it is reasonable to conclude, based on the measurable weight changes on the specimen collection membrane filter that this subject yielded MAS.

Based on the findings from currently accepted standards of medical care, all breasts examined were normal in that none demonstrated any clinically significant abnormality on either CBE or mammography. These findings were consistent with the findings on MAS in which no specimen test result suggested any clinically significant abnormality (defined as Class III or IV cytological finding).

Patients being considered for cytopathologic evaluation of specimens collected using the MASCT System should undergo concurrent evaluation using currently accepted standards of care (for example, testing performed in accordance with National Comprehensive Cancer Network $\left(\mathrm{NCCN}^{\circledR}\right)$ guidelines [8]. Decisions regarding the appropriateness of cytopathologic testing should be based on the physician's assessment of the individual patient's history, clinical presentation, and other factors that he/she deems to be appropriate.

The device is intended to be used to collect specimens for cytopathology testing in addition to the testing performed in accordance with the currently accepted standards of medical care (for example, testing performed in accordance with NCCN guidelines). The intended patient population is, therefore, any woman for whom NAF cytology testing is considered to be beneficial by her physician.

Therefore, the results of MAS testing in this subject series accords with the findings of the current "gold standard" methods of evaluating breast health, i.e., the clinical breast exam plus mammogram.

\section{CONCLUSION}

Based on this clinical study, the Mammary Aspiration
Specimen Cytology Test is safe and effective for use in the collection of mammary aspirate specimens for laboratory cytopathological testing.

\section{ACKNOWLEDGEMENTS}

The authors wish to thank Harris Michael, M.S. for monitoring the study and Weining Liang, M.D., Rose Linda Grummer, R.N., Peter Aprile, R.Ph, Cynthia Price, B.S. and Anthony Sileno, M.S., for assistance in the conduct of the study. This study was financially supported by a clinical trial grant from Nastech Pharmaceutical, Inc., Hauppauge, New York.

\section{REFERENCES}

[1] Petrakis, N.L. (1993) Studies on the epidemiology and natural history of benign breast disease and breast cancer using nipple aspirate fluid. Cancer Epidemiology, Biomarkers \& Prevention, 2, 3-10.

[2] King, E.B., Chew, K.L., Petrakis, N.L. and Ernster, V.L. (1983) Nipple aspirate cytology for the study of breast cancer precursors. Journal of the National Cancer Institute, 71, 1115-1121.

[3] Byrd, B.J. (1974) Standard breast examination. American Cancer Society, New York.

[4] American College of Radiology (1995) Breast imaging reporting and data system (BI-RADS). 2nd Edition, American College of Radiology, Reston.

[5] Kent, J.C., Mitoulas, L.R., Cregan, M.D., Geddes, D.T., Larsson, M., Doherty, D.A. and Hartmann, P.E. (2008) Importance of vacuum for breast milk expression. Breastfeeding Medicine, 3, 11-19. doi:10.1089/bfm.2007.0028

[6] Henson, D.E., Oberman, H.A., Hutter, R.V.P. and College of American Pathologists (1997) Practice protocol for the examination of specimens removed from patients with cancer of the breast. Archives of Pathology \& Laboratory Medicine, 121, 27-33.

[7] King, E.B., Chew, K.L., Petrakis, N.L. and Ernster, V.L. (1983) Nipple aspirate cytology for the study of breast cancer precursors. Journal of the National Cancer Institute, 71, 1115-1121.

[8] NCCN (2013) Clinical practice guidelines in oncology breast cancer screening and diagnosis. Version I. www.NCCN.org 